\title{
Pathogen-Sensing, Regulatory T Cells, and Responsiveness-Tuning Collectively Regulate Foreign- and Self-Antigen Mediated T-Cell Responses
}

\author{
William E. Paul, Joshua D. Milner, and Zvi Grossman \\ Laboratory of Immunology and Laboratory of Allergic Diseases, National Institute of Allergy and \\ Infectious Diseases, National Institutes of Health, Bethesda, Maryland 20892-1892 \\ Correspondence: wpaul@niaid.nih.gov
}

\begin{abstract}
The concept that induction of T-cell-dependent immune responses requires both T-cell recognition of an epitope and recognition by the innate immune system of pathogen-associated molecular patterns needs to be extended to include the contribution of regulatory $\mathrm{T}$ cells and of responsiveness-tuning. Here we develop the hypothesis that both pathogen sensing and regulatory $\mathrm{T}$ cells act on antigen-presenting dendritic cells (DCs) to determine whether the DCs will be competent to activate $\mathrm{T}$ cells with cognate receptors. Tregs that recognize self-peptide/major histocompatibility complexes (MHCs) on the DC in question will serve to inactivate that DC, whereas sensing of pathogens or innate "danger" signals by the DC will oppose the action of the Tregs. The responsiveness of the T-cell compartment is further controlled by activation-threshold tuning in which responsiveness is adjusted to the strength of recurrent stimulation of T cells by self-peptide/MHCs in the periphery. Thus, a robust T-cell response depends on T-cell recognition of a peptide/MHC presented by a competent DC, competence depending on interactions with Tregs and the degree of innate stimulation through pathogen sensing, and on a combined TCR and accessory-signaling strength of stimulation that exceeds the activation threshold of the $\mathrm{T}$ cells, determined by its responsiveness tuning.
\end{abstract}

Twenty-four years ago, in his Introductory Lecture at the 54th Cold Spring Harbor Symposium on Quantitative Biology, Charles A. Janeway, Jr. created the modern synthesis of immunology by linking pathogen-sensing, the innate immune response, and the induction of adaptive immunity (Janeway 1989). The reverberations of that lecture, "Approaching the Asymptote? Evolution and Revolution in Immunology," are still being felt today, much as radiation emanating from the Big Bang continues to be detected. One of us (WEP) was Charlie's mentor for five years at the National Institutes of Health (NIH). In this regard, the Talmudic epigram, "From my teachers I have learned much, from my colleagues still more, but most of all, from my students," is particularly apt.

In this Closing Lecture to the "once-a-decade" forays of the Quantitative Biology Symposia into the field of immunology, we will be presumptuous enough to hope that we can extend Charlie's dicta even a little by taking advantage of many ideas that have been already introduced by our group and others, particularly Charlie's student Ruslan Medzhitov.

\section{THE JANEWAY SYNTHESIS}

Charlie's central idea, the immunologist's "dirty little secret," was that foreign antigens fail to elicit robust responses unless adjuvants are provided, acting as surrogates for pathogenic microbes, or in Polly Matzinger's hypothesis (Matzinger 1994), for endogenous danger signals.
What Charlie said is the general experience we all have. Indeed Charlie's synthesis was anticipated by the work of the first incumbent of the position of Chief of the Laboratory of Immunology, Jules Freund, who introduced what has been the most widely used of the potent experimental adjuvants, complete Freund's adjuvant, and showed how central it was to achieve a robust response (Freund et al. 1953).

\section{LYMPHOPENIA AND ADJUVANT EFFECTS}

And yet there is at least one situation in which exogenous adjuvants are not required for robust antigen-driven expansion of specific CD4 T cells. That is, when the immunized recipient is lymphopenic. For example, transfer of CD4 T cells from a 5C.C7 T-cell receptor transgenic donor, specific for a pigeon cytochrome $\mathrm{C}$ (PCC) sequence, to a Rag2 $2^{-/-}$B10.A recipient and challenge with PCC peptide without adjuvant results in the striking expansion of the transferred cells; indeed, these cells expand to a much greater extent than do the same cells transferred to an intact recipient that received antigen plus an adjuvant, and almost as robustly as if lipopolysaccharide (LPS) were coadministered with peptide to the lymphopenic recipient (Fig. 1) (J Quiel and JD Milner, unpubl.).

What are the fundamental differences between the lymphopenic and the conventional recipient of 5C.C7 cells or for that matter the normal mouse undergoing a physiological immune response? There are obviously two, both of 


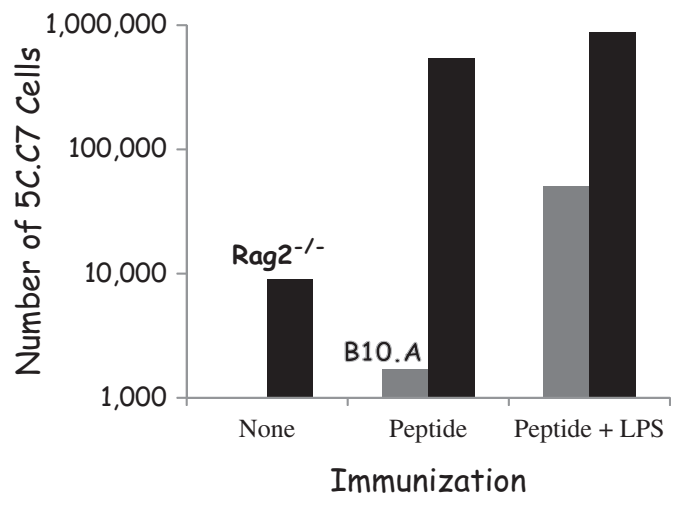

Figure 1. Equal numbers of 5C.C7 cells were transferred to wild-type and Rag2 ${ }^{-/-}$B10.A recipients that were immunized with pigeon cytochrome $c$ peptide with or without LPS. The number of 5C.C7 in lymph nodes was measured $7 \mathrm{~d}$ later. (J Quiel and J Milner, unpubl.)

which need consideration. The first is the absence of other lymphocytes, both conventional and Tregs that could profoundly affect the behavior of introduced CD4 T cells. The second is the absence of cells that control intestinal microbiota, which might allow microbial translocation and "spontaneous" innate sensing to occur.

With Booki Min, we considered the possibility that repertoire complexity of conventional CD4 $\mathrm{T}$ cells resident in an animal might control the capacity of transferred cells to proliferate. It was known that Rag1 or Rag2 knockout Tcell receptor transgenic mice, which have but a single type of CD4 T cell, allow transferred cells to undergo proliferation, much as mice completely lacking $\mathrm{T}$ cells do. This suggested that $\mathrm{T}$-cell receptor complexity would be important in determining the proliferation of transferred polyclonal cells. Mice were prepared that had similar number of CD4 T cells but in which the complexity of the T-cell receptor repertoire varied. This was done by transferring increasing number of naïve CD4 $\mathrm{T}$ cells to $\mathrm{Rag} 2^{-/-}$recipients and assessing their repertoire complexity by immunoscope analysis when they had reached equilibrium. The complexity of TCR $\beta$ chains increases as the number of transferred cells increase although, at equi- librium, the absolute number of these cells is the same (Fig. 2) (Min et al. 2004). When a subsequent population of polyclonal naïve CD4 $\mathrm{T}$ cells was transferred to these recipients, the expansion and proliferation of the cells diminished as the number of originally transferred cells and thus their receptor complexity increased (Fig. 3). We postulated that cells of similar specificity, probably for selfpeptide/MHCs, and of similar phenotype regulated one another's capacity to expand. Indeed, such competition/ inhibition based on T-cell receptor specificity does occur as shown in various settings by Antonio Freitas and his colleagues (Almeida et al. 2005) and more recently by Juan Quiel et al. (2011) and by Nevil Singh and Ron Schwartz et al. 2012. In the latter studies, conventional $\mathrm{T}$ cells appeared to inhibit each other, independently of whether Tregs were present or not.

Although we originally interpreted the Min et al. experiment in terms of the capacity of cells of the same phenotype to regulate one another's expansion, we subsequently realized that the regulation of expansion might be controlled primarily by Tregs and that the complexity of the Treg repertoire might be the critical determinant in whether the conventional $\mathrm{T}$ cells would expand and to what extent. To study whether changes in receptor complexity of Tregs could account for the differential capacity of newly transferred cells to expand, CD45.2 Rag2 ${ }^{-/-}$ mice received either $0,3 \times 10^{4}$ or $3 \times 10^{5}$ sorted $\mathrm{CD} 25^{+}$ CD45.1 CD4 T cells or CD25 ${ }^{+}$CD45.2 CD4 T cells intravenously. Ten weeks after primary $\mathrm{CD} 25^{+} \mathrm{CD} 4 \mathrm{~T}$-cell transfer, when the total number of initially transferred cells was shown to be similar (using recipients of CD45.1 cells), 1 million CFSE-labeled CD45.1 CD25 CD4 T cells were transferred into recipients of CD45.2 cells. Upon killing 1 wk later, both groups of mice that received Tregs had many fewer conventional CD4 T cells than did the mice that only received conventional CD4 T cells. Those that received Tregs derived from the group that had initially received 300,000 cells showed even greater suppression of conventional CD4 T-cell expansion than the group that received Tregs derived from the mice that received 30,000 Tregs; in fact, in the recipients of Tregs derived from mice that had initially received 300,000 cells, the conventional CD4 T cells had hardly

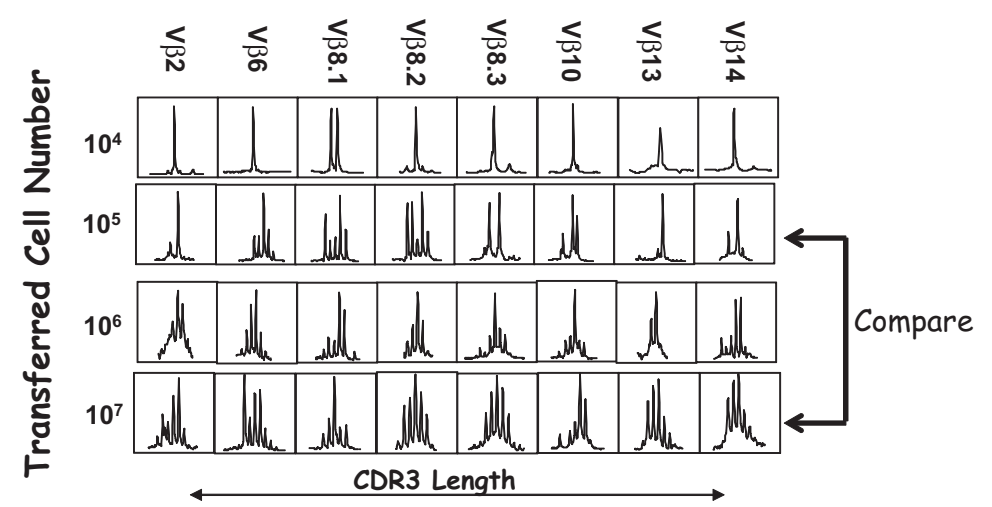

Figure 2. Different numbers $\left(10^{4}-10^{7}\right)$ of Ly5.2 CD4 T cells were transferred into Rag2 $2^{-/-}$mice. Two months later, repertoire complexity of the transferred cells was determined by immunoscope analysis. (Adapted from Min et al. 2004.) 


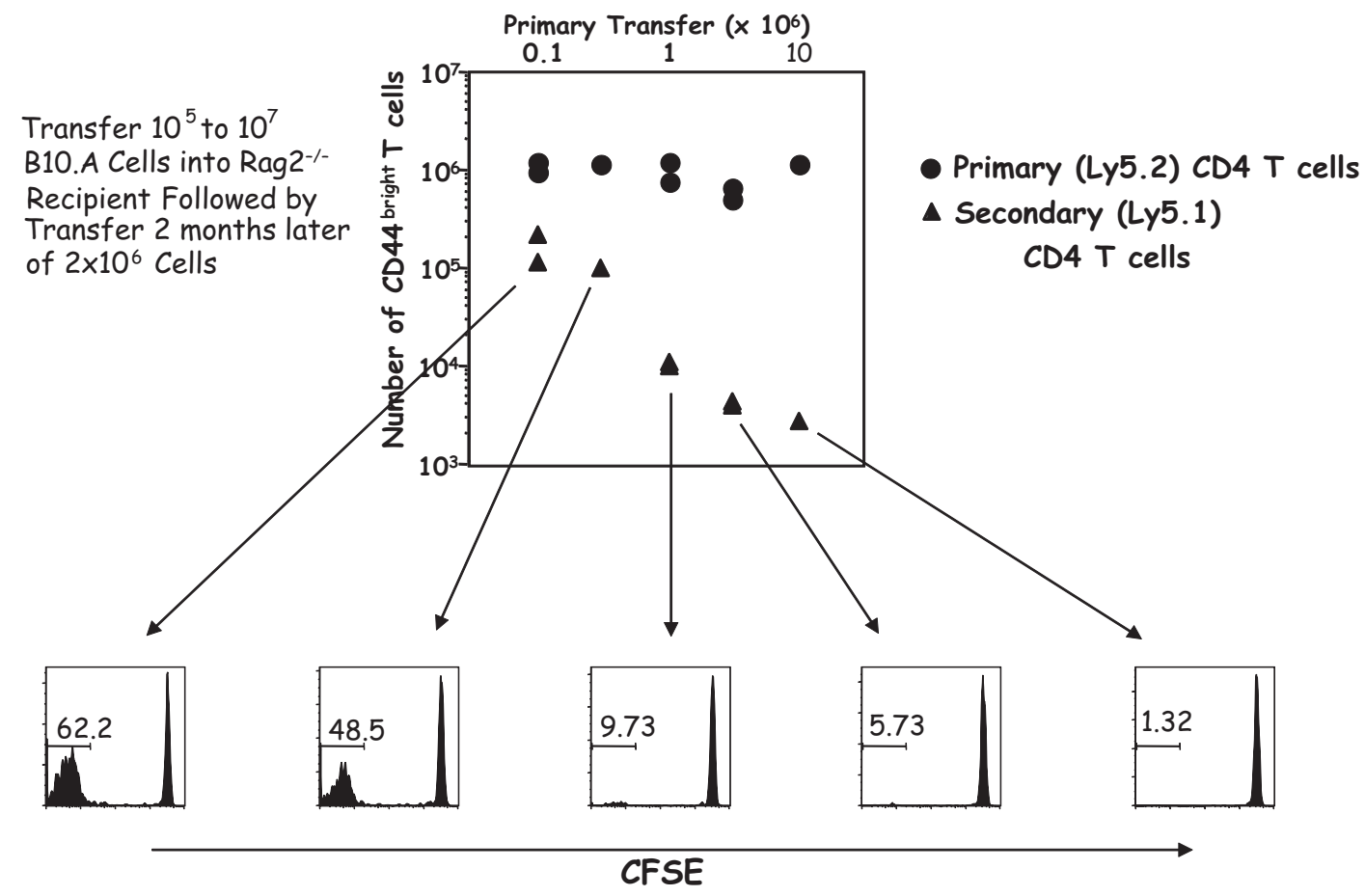

Figure 3. Rag $2^{-/-}$recipients as described in the legend of Figure 2 received $2 \times 10^{6}$ CFSE-labeled Ly5.1 naïve CD4 T cells. Seven days after transfer, the total number of CD44 $4^{\text {bright }}$ Ly5.1 CD4 T cells and CFSE profiles from lymph nodes and liver was determined. (Adapted from Min et al. 2004.)

divided at all (Fig. 4) (Milner et al. 2007). Thus, lymphopenia-induced proliferation is suppressed by Tregs and the number of "founder" Tregs, and thus the complexity of the TCR repertoire of the Tregs transferred together with the conventional CD4 T cells determines the efficacy of the Tregs in their control of lymphopenia-induced proliferation.

The importance of Tregs in determining expansion of conventional CD4 $\mathrm{T}$ cells is showed by the consequences of acute depletion of Tregs. G. Punkosdy and E. Shevach (unpubl.) administered diphtheria toxin (DT) to transgenic mice in which the expression of the DT receptor is under the control of a bacterial artificial chromosome (BAC)encoded Foxp 3 promoter. In these mice, Tregs are acutely eliminated and a rapid and massive increase occurs in the frequency of $\mathrm{CD} 44^{\text {bright }} \mathrm{CD} 4 \mathrm{~T}$ cells and in the proliferative rate of these cells, very reminiscent of the behavior of transferred CD4 T cells in a lymphopenic recipient. The expansion of these CD4 $\mathrm{T}$ cells is partially blocked by antibodies to class II MHC molecules suggesting that it depends in part upon TCR recognition of peptide/MHCs and in part on cytokine-mediated memory-phenotype $\mathrm{T}$-cell expansion. These results provide very persuasive evidence that in the absence of Tregs, TCR-driven CD4 T-cell expansion occurs without need for adjuvant administration. Alexander Rudensky and his colleagues reported a similar experiment with a differently constructed DT-sensitive mouse (Kim et al. 2009). They showed that when Tregs are acutely depleted, dendritic cells (DCs) become activated. However, this activation depends on the presence of conventional CD4 T cells, implying that
DC activation is normally restrained by Tregs but can be induced by conventional $\mathrm{T}$ cells even in the absence of exogenous adjuvants if Tregs are absent.

The issue of the possible role of microbial translocation and the consequent activation of the innate immune system in the antigen-driven expansion of specific cells in lymphopenic and Treg-depleted mice needs to be directly tested and the data obtained could obviously lead to a reinterpretation of some of these results.

\section{PROPOSITION 1: Tregs KEEP DCS “OFF"' BUT ADJUVANTS COOPERATE WITH T CELLS TO TURN THEM “ON"}

According to proposition 1, antigen-driven expansion of specific CD4 T cells is normally restrained by Tregs. Cell-based suppression/competition mechanisms, particularly the action of Tregs, maintain DCs in an "off" state. The action of adjuvants is to overcome these restraints and to allow CD4 T cells to promote DCs to an "on" state despite the presence of Tregs. The fundamental idea of the opposing actions of pathogen-sensing and Tregs is not new. Pesare and Medzhitov presented it in a very influential 2003 Science paper (Pasare and Medzhitov 2003), but in our view this notion is of sufficient importance to bear repetition and expansion, particularly in view of advances in the understanding of the mode of action of Tregs. The concept of opposing actions on the same DC forms the central element of the integrative outlook we wish to develop. 


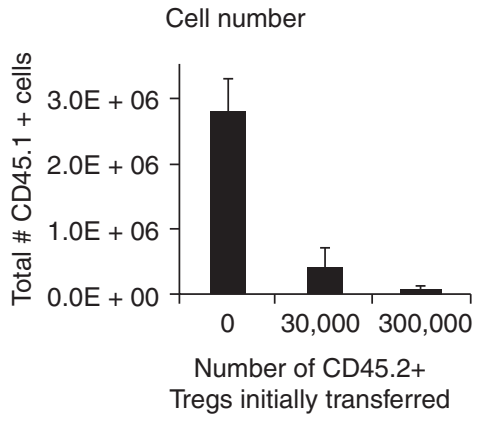

Cell proliferation

Division of CD45.1 + CD25-transferred cells

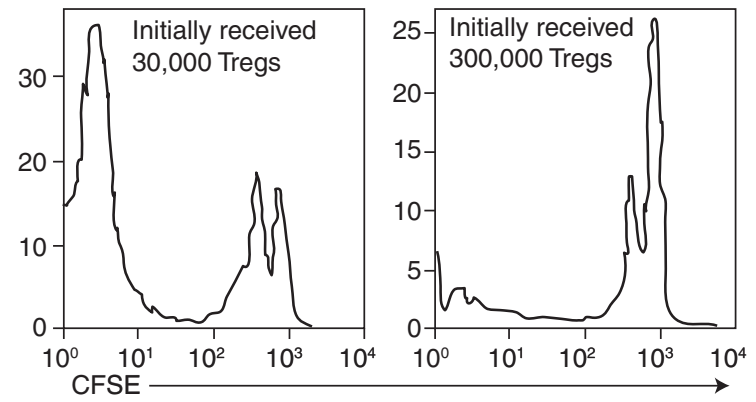

Figure 4. CD45.2 $\mathrm{Rag}^{-/-}$mice received either $0,3 \times 10^{4}$ or $3 \times 10^{5} \mathrm{CD} 25^{+} \mathrm{CD} 45.1 \mathrm{CD} 4 \mathrm{~T}$ cells or CD25 ${ }^{+} \mathrm{CD} 45.2 \mathrm{CD} 4 \mathrm{~T}$ cells intravenously. Ten weeks after primary CD $25^{+} \mathrm{CD} 4 \mathrm{~T}$-cell transfer, when the total number of initially transferred cells was shown to be similar (using recipients of CD45.1 cells), 1 million CFSE-labeled CD45.1 CD25 ${ }^{-}$CD4 T cells were transferred into recipients of CD45.2 cells, and lymph nodes were harvested 1 wk later. Cell numbers and CFSE profiles of transferred CD45.1 $\mathrm{T}$ cells are shown. (Adapted from Milner et al. 2007.)

We restate this concept in more mechanistic terms as follows. The role of Tregs is to keep DCs "off." In the absence of Tregs, DCs will turn on if they present peptide/MHCs to T cells capable of recognizing those complexes, presumably due to the expression of CD40 ligand (CD40L) on the T cells and its interaction with CD40 on the DC (Fig. 5A-C). This turn "on" of DCs would be blocked by Tregs that themselves interact with the same DCs. Tregs and conventional CD4 T cells need not interact simultaneously with DCs; rather, the Tregs may serve to inactivate the DCs so that they are unresponsive to subsequent interaction with conventional CD4 T cells. However, adjuvants or strong exogenous pathogen-sensing will overcome the Treg-mediated restraint on DC activation and allow $\mathrm{T}$ cells interacting with those DCs to expand and differentiate even if Tregs have acted on the antigen-presenting DCs.

Our colleague Ethan Shevach (2011) has shown that in vitro, the turn "on" of antigen-pulsed DCs is enhanced by interaction with antigen-specific conventional CD4 T cells but this turn "on" is inhibited by the presence of iTregs specific for the same antigen (Fig. 6). Thus, we would argue that in a lymphopenic, Treg-depleted environment either a subset of self-peptide/MHCs or translocated microbial antigens, or both, may trigger activation and expansion of specific T-cells but if Tregs capable of interacting with the same DCs that had stimulated the conventional CD4 T cells were present, they would block such activation.

We recognize that characterizing DCs as in "on" or "off" states is an oversimplification but for clarity, we will use this formulation in the course of this presentation. In addition, although we have emphasized the role of Tregs in restraining the activation of DCs, it is clear that Tregs have a variety of other functions. One could argue that once evolved for the purpose of controlling DC activation, Tregs took on many other functions that are now of great importance.

To recapitulate, in settings in which Tregs are absent or in which they have very limited repertoires, those antigen-specific CD4 $\mathrm{T}$ cells that encounter their cognate foreign antigen or in some instances, self-peptide/MHCs displayed by DCs, will turn these DCs "on." These $\mathrm{T}$ cells will then expand even without the induction of an inflammatory state mediated by the pathogen sensing mechanisms of the innate immune system or perhaps with a minimal level of activation of the innate immune system resulting from microbial translocation. However, when the number and repertoire of Tregs are sufficient to maintain the great majority of DCs in an "off" state, exogenous adjuvants or potent pathogen sensing will be essential for CD4 T-cell expansion and differentiation.

\section{LYMPHOPENIA-INDUCED PROLIFERATION}

The "on"/“off” concept also governs lymphopenia-induced polyclonal proliferation. The latter is not the unregulated expansion of all cells transferred into an "empty" host but rather represents the capacity of a relatively small

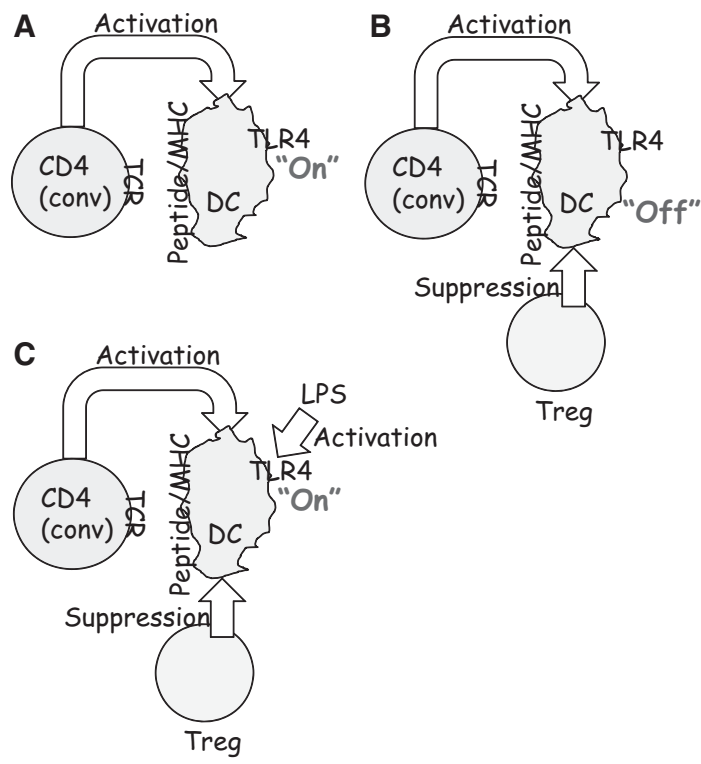

Figure 5. $(A-C)$ Schematic depiction of the "on"/"off" regulation of DC by the opposing actions of Tregs and LPS, with T cells recognizing peptide/MHCs on DC playing a critical role in the activation of DC. 


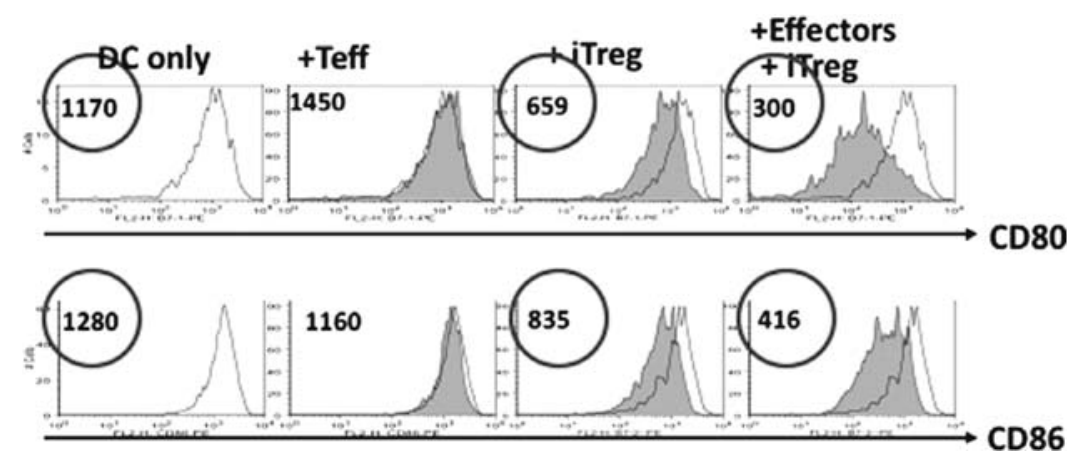

Figure 6. Splenic DCs pulsed with peptide, washed, and cultured for $18 \mathrm{~h}$ alone, or in the presence of antigen-specific Teff, iTregs, or a mixture of Teff and iTregs. The expression of CD80 and CD86 was quantitated by FACS analysis. The numbers in the circles indicate the mean fluorescence intensity. (Reprinted, with permission, from Shevach 2011, (C) Elsevier.)

subset of memory-phenotype conventional CD4 T cells, probably less than $10 \%$, and an even smaller proportion of naïve cells, to expand in the lymphopenic setting. This occurs as a result of TCR-based recognition of peptide/MHCs displayed by DCs that are allowed to turn "on." Evidence for this conclusion is provided by two experiments we have done and by the work of others. Souheil Younes transferred a set of CD $44^{\text {bright }}$ CD4 T cells into Rag $2^{-1-}$ recipients and showed that their expansion in those recipients was blocked by anti-class II MHC antibodies, implying it was TCR driven (Fig. 7) (Younes et al. 2011). We then measured the number of distinct CDR3 sequences among the CD4 $\mathrm{T}$ cells expressing a particular $\mathrm{V} \beta / \mathrm{J} \beta$ combination in the recipient. Of 42 CDR3 sequences in transferred cells that had not divided in the lymphopenic recipient, 39 were unique, whereas of 42 sequences among the cells that had undergone several rounds of division, there were only three unique sequences, each represented multiple times. Similarly, using "immunoscope" analysis, we measured the frequency of distinct $\mathrm{V} \beta$ types among naïve $\mathrm{CD} 4 \mathrm{~T}$ cells that expanded when transferred to a lymphopenic recipient. These results implied that only $\sim 3 \%$ of the transferred naïve cells underwent lymphopenia-induced proliferation (Fig. 2).

These results are in keeping with the paucity of TCR transgenic CD4 $\mathrm{T}$ cells that proliferate when transferred to lymphopenic recipients. Mandl et al. (2013) have compared the level of CD5 expressed by different TCR transgenic CD4 $\mathrm{T}$ cells to their expansion potential in lymphopenic recipients and found an excellent correlation (Fig. 8). In general, CD5 levels are directly associated with
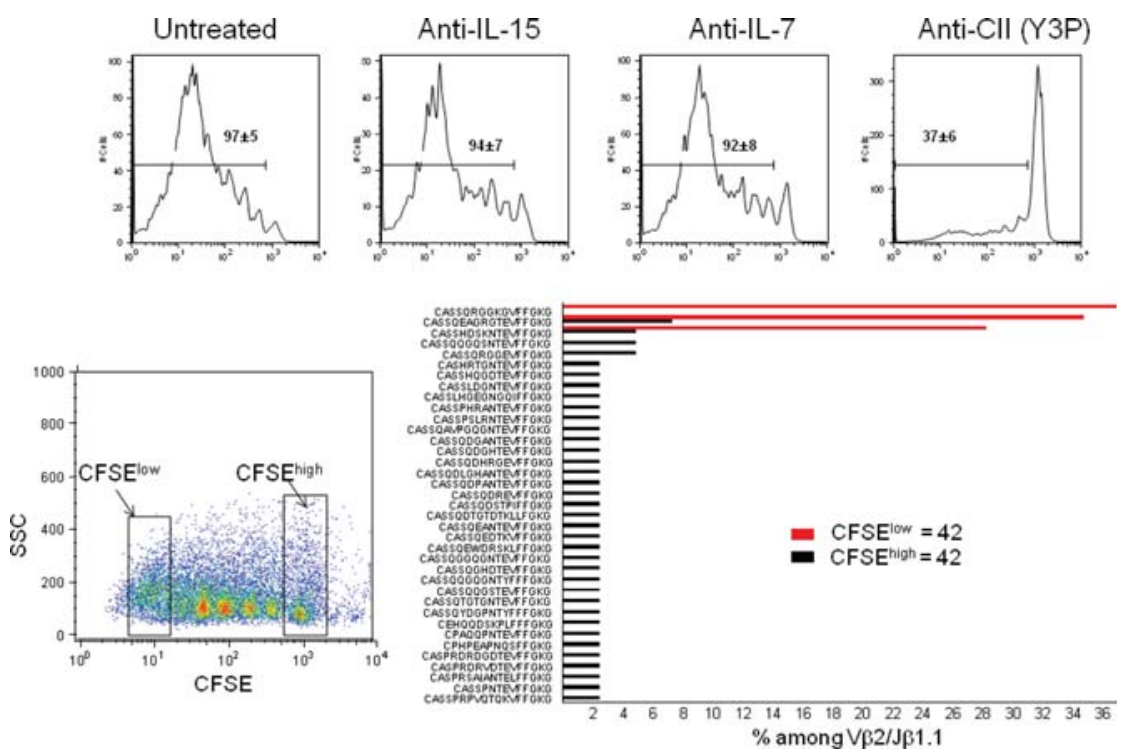

Figure 7. One million CFSE-labeled memory phenotype (MP) CD4 T cells were transferred into Rag $2^{-/-}$mice that received antiIL-7, anti-IL-15, or Y3P (anti-class II) or were untreated. On day 6 flow cytometric analysis of CFSE dilution was performed. In a separate experiment, 1 million CFSE-labeled MP CD4 T cells were transferred into Rag $2^{-}{ }^{-}$recipients. On day 3 , CFSE low and high cells were sorted, followed by PCR amplification using V 32 and J $\beta 1.1$ primers. CDR3 sequences were plotted according to the frequency with which they appeared among the 42 sequences obtained from CFSE low and high V $\beta 2 / \mathrm{J} \beta 1.1$ cells, respectively. (Reprinted from Younes et al. 2011.) 


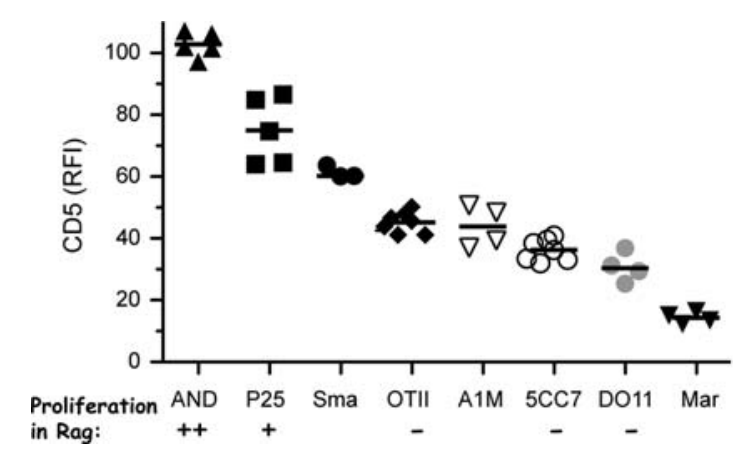

Figure 8. CD5 surface expression on various TCR transgenic $\mathrm{CD} 4 \mathrm{~T}$ cells relative to that of polyclonal $\mathrm{CD}^{+}{ }^{+} \mathrm{T}$ cells from mice of the same genetic background. (Adapted from Mandl et al. 2013, (C) Elsevier.)

strength of interaction of CD4 T cells with their selecting self-peptide/MHC (Azzam et al. 1998). These results suggest that in the absence of their cognate foreign antigens, only those cells with relatively high affinity for selfpeptide/MHCs (or possibly peptide/MHCs involving peptides from commensal organisms) can expand when freed of control by Tregs, that is when confronted by DCs that turn "on" with no opposing "off" signal.

What happens when mice are infected with pathogens that potently activate the innate immune system? Y. Belkaid and her colleagues (Oldenhove et al. 2009) and J. Zhu and his group (unpubl.) have independently shown that mice infected with Toxoplasma gondii show rapid diminution in the frequency of Tregs, their numbers falling almost 10 -fold by day 7-8 of infection (Fig. 9). In parallel, there was a great increase in the frequency of $\mathrm{CD}^{+} \mathrm{T}$ cells capable of producing IFN- $\gamma$ or expressing a memory phenotype, as exemplified by being CD $44^{\text {bright }}$. Although some of these cells are surely specific for
T. gondii antigens, we argue that many are not, reflecting CD4 T cells that have been stimulated to expand by DC that are no longer controlled by Tregs. This suggests a more complex situation in which $\mathrm{T}$ cells expand in response to both an "adjuvant" effect and a release from Treg control. Immune responses occur when a preexisting balance between the two regulators of DC activity is transiently perturbed. What fraction of acute infections cause such Treg depletion needs to be assessed but it has been shown to be the case for Listeria monocytogenes and vaccinia as well as T. gondii (Benson et al. 2012) and has been attributed to limitation in the availability of IL-2 due to expansion of conventional CD4 T cells.

\section{CONVENTIONAL CD4 T CELLS AND Tregs HAVE DIFFERENT TCR REPERTOIRES}

Conventional CD4 T cells are positively selected in the thymus by virtue of the strength of the interaction of their TCRs with one or more self-peptide/MHCs displayed by cortical thymic epithelial cells (cTECs). The CD4 T cells so selected form the naïve repertoire from which cells responding to particular foreign-peptide/MHCs will be derived. As we stated above, such peripheral antigendriven responses require that the antigen-presenting DCs be in an "on" state which is achieved either as a result of the absence of Tregs that can act on those DCs or, even in the presence of such Tregs, if a potent adjuvant has been introduced. A major advantage provided by selfpeptide/MHC selection in the thymus is that it provides a broad population of naïve CD4 T cells that can recognize and respond to foreign peptide/MHCs.

In contrast, not only are thymus-induced Tregs selected on self-peptide/MHCs in the thymus, it is likely that they predominantly mediate their function in the periphery
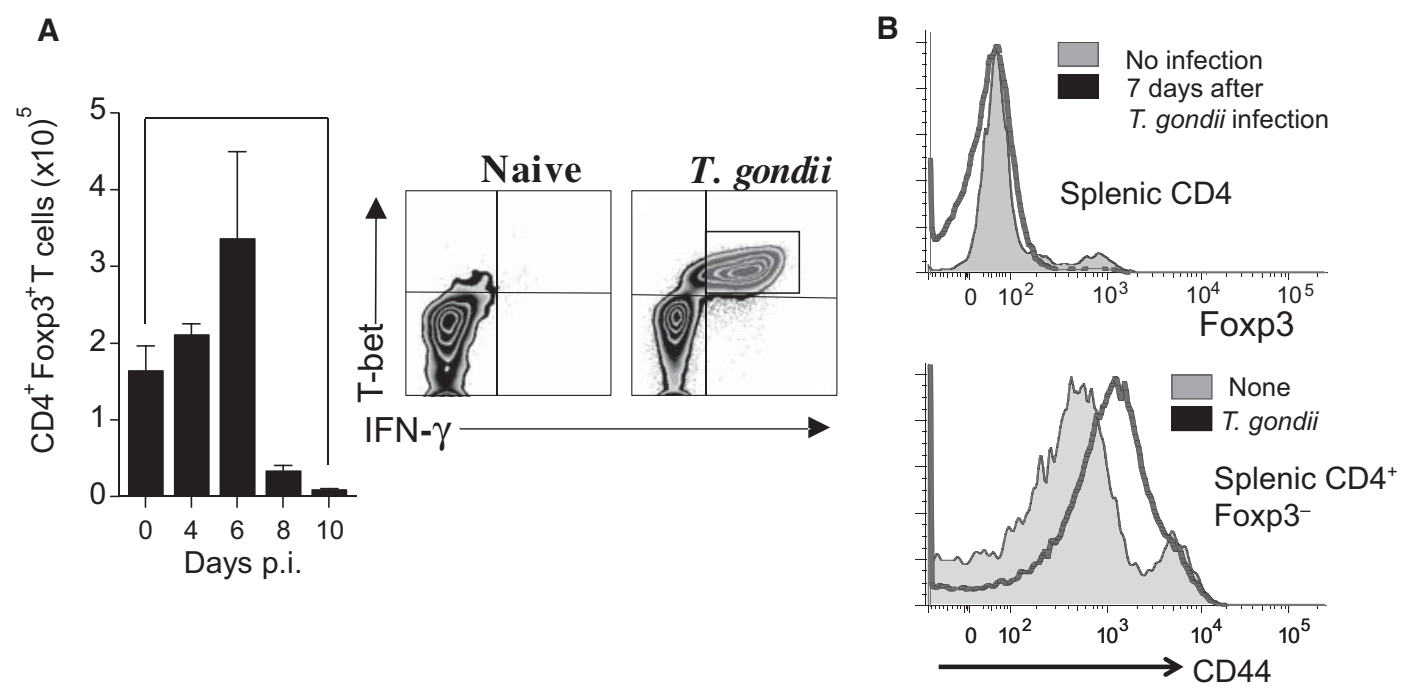

Figure 9. (A) Absolute number of TCR- $\beta^{+} \mathrm{CD}^{+}{ }^{+} \mathrm{Foxp} 3^{+}$cells in mesenteric lymph nodes between day 0 and 12 of infection with Toxoplasma gondii and T-bet and IFN- $\gamma$ expression in naïve, esenteric lymph nodes or in mesenteric lymph nodes on day 8 of infection. (B) Splenic Foxp ${ }^{+}$and CD $44^{\text {bright }}$ Foxp3- cells $7 \mathrm{~d}$ after T. gondii infection. (A, Adapted from Oldenhove et al. 2009, (C) Elsevier; $B$, courtesy of J.-F. Zhu, Laboratory of Immunology, NIAID.) 
by recognizing the same or related self-peptide/MHCs (Hsieh et al. 2004). This provides the evolutionary advantage for their affinity for the selecting peptide/MHC to be high, because that complex will be the Tregs" "cognate" antigen in the periphery. It appears that induction of the Treg phenotype through such high-affinity interaction evolved as an alternative to thymic deletion.

\section{PROPOSITION 2: INDIVIDUAL DCS PRESENT SELECTED SETS OF PEPTIDE/MHCs}

Because it appears most likely that a principal mode of action of Tregs is to inhibit DCs or, as we suggested above, to prevent CD40/CD40L interactions to move them to an "on" state, the main need is that the Tregs recognize and "inhibit" those DCs with which conventional CD4 T cells can interact. There is no need for the Treg and the conventional CD4 T cell to be specific for the same antigen, only for the DC to express both a peptide/ MHC recognized by the Treg (in this case the selecting peptide or a related self-peptide/MHC) and a peptide/ $\mathrm{MHC}$ recognized by the conventional CD4 $\mathrm{T}$ cell (either self- or a foreign-peptide/MHC).

If each DC was capable of presenting a very broad range of self-peptide/MHCs, Tregs might be expected to be highly efficient, on a per cell basis, in controlling autoimmune responses, irrespective of their repertoire complexity. Under such circumstances, any "self-reactive" Treg should be able to suppress almost any DC. But we showed earlier that Tregs of limited repertoire were less effective in controlling the expansion of polyclonal CD4 $T$ cells in a lymphopenic environment than were Tregs of a broad repertoire (Fig. 4). Hence we introduce proposition 2. Each individual DC effectively presents only a limited number of self-peptide/MHCs and the set of self-peptide/ MHCs any particular DC presents is randomly or at least partially randomly determined. Such specialization could have broad significance in the regulation of immune responses (Grossman et al. 2004).

Tregs and conventional CD4 $\mathrm{T}$ cells specific for the same peptide/MHC will of necessity be capable of interacting with the same DCs. This would not generally be the case when the Tregs and the conventional CD4 T cells recognize different peptide/MHCs if each DC presents only a limited set of peptide/MHCs. However, if the repertoire of the Treg population is broad enough, as it should be in intact animals or in recipients of large numbers of polyclonal Tregs, then there should be Tregs capable of recognizing and regulating most if not all the DCs presenting a given peptide/MHC recognized by a particular conventional CD4 T cell. This would account for the result that polyclonal Tregs of limited repertoire are less effective than the same number of Tregs of broad repertoire in controlling lymphopenia-induced proliferation. A Treg population with a limited TCR repertoire would be capable of regulating the activation of a smaller proportion of DCs than would a Treg population that had a large TCR repertoire even if the number of Tregs were the same.

\section{EVIDENCE FOR DC-TARGETED REGULATION AND SELECTIVE PEPTIDE PRESENTATION}

What is our evidence suggesting such DC "specialization" in the presentation of self-peptide/MHCs? In the case of DCs presenting foreign peptide/MHCs, we recently argued that specialization could account for the specificity of T-cell inhibition of T-cell expansion. In these experiments, we first showed that small number of naïve TCR transgenic CD4 T cells expand, on a per cell basis, in response to their cognate antigen, to a much larger extent than a large number of the same cells. For example, three 5C.C7 cells in a conventional B10.A recipient expand more than 1000 -fold in $7 \mathrm{~d}$ in response to immunization with PCC and LPS. Three hundred 5C.C7 cells expand $\sim 100$-fold and 30,000 5C.C7 cells expand only $\sim 10$-fold upon antigen challenge (Fig. 10) (Quiel et al. 2011). This could be interpreted as indicating that a large number of 5C.C7 cells diminished the degree of expansion of a small number of the same cells upon antigen stimulation. This provides an example of the inhibition of expansion mediated by cells of the same phenotype as the cells being inhibited. This phenomenon has been shown to hold for all the TCR transgenic CD4 T cells we tested including A1M, OT-II and Marilyn.

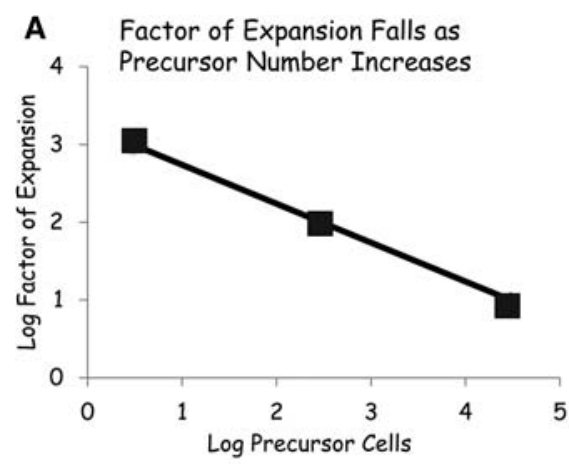

B Transfer Marilyn and/or OT-2 Cells,

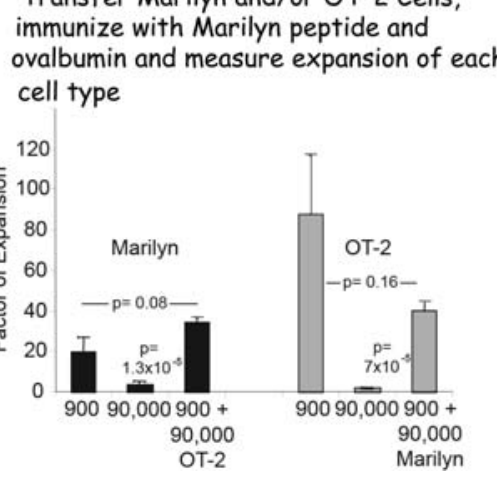

Figure 10. (A) Varying numbers of 5C.C7 cells were transferred to normal B10.A recipients that were then immunized with pigeon cytochrome $\mathrm{C}$ peptide and LPS. The number of 5C.C7 in lymph nodes was measured 7 d later. (B) 900 or 90,000 OT-2 and/or Marilyn cells were transferred to male C57BL/6 mice that were then immunized with OVA peptide. The number of OT-2 and Marilyn cells was measured $7 \mathrm{~d}$ later. (Adapted from Quiel et al. 2011.) 
We found that although a small number of OT-II cells were inhibited in their expansion by a large number of the same cells, they showed little or no diminution in their expansion in the presence of a large number of Marilyn TCR transgenic cells, specific for a Y chromosome-encoded peptide/MHC and responding to their cognate antigen presented by male CF57BL/6 mice. A large number of Marilyn cells did inhibit the expansion of a small number of Marilyn cells. The recipient mice received ovalbumin and, being males, expressed the $\mathrm{Y}$ chromosome-encoded peptide. Thus, inhibition by cells of the same phenotype was antigen-specific.

Removal of cognate peptide/MHCs from the DCs by the $\mathrm{T}$ cells upon binding these complexes would explain our results. Indeed, evidence has been provided that the interaction of CD8 T cells with antigen-presenting DCs results in such removal (Kedl et al. 2002). But such an explanation of our results would predict a strong dependence of the magnitude of expansion on peptide concentrations used in immunization, and such dependence was not found (Quiel et al. 2011). We thus explained the specificity of the inhibition observed in our experiments by the proposed preferential expression of peptides from ovalbumin and the Y peptide on different DCs. Direct evidence for a negative feedback operating at the level of the DCs, whereby interacting T cells can diminish the antigen-presentation capacity of the DC, has been reported (Vendetti et al. 2000).

The peptide/MHC removal model is even less likely to explain the action of Tregs in the control of DC stimulation of conventional CD4 $\mathrm{T}$ cells, because the receptor repertoires of Tregs and conventional CD4 $\mathrm{T}$ cells are different (Hsieh et al. 2004). Indeed, to account for the differential efficacy of Treg populations of complex and simple repertoires in control of CD4 T-cell lymphopeniainduced proliferation through removal of the peptide/ MHCs by Tregs, the repertoires would need to be essentially the same. Adopting the model in which the DC is the Treg target, the key issue is the pattern of expression of self-peptide/MHCs. The expression of only a subset of self-peptide/MHCs at a high-enough frequency on any particular DC to activate Tregs with cognate TCRs could be accounted for by stochastic variability in intracellular content of various self-proteins.

Recapitulating, DC specialization in peptide/MHC expression would explain why Tregs of limited repertoire would be less effective than the same number of Tregs of broad repertoire in keeping most DCs in an "off" state. Limited repertoire Treg populations would be unable to interact with at least some DCs, those that by chance do not display self-peptide/MHCs that some Tregs could recognize. Such DCs could achieve an "on" state when they present foreign-peptide/MHCs to specific CD4 $T$ cells and could then stimulate responses to such foreign peptide/MHCs in the absence of adjuvants and even to some self-peptide/MHCs to which tolerance has not been adequately established. We note that DCs could escape suppression by Tregs even if they do display the appropriate self-peptide/MHCs but only at low concentrations, because the frequency and strength of the interaction be- tween the two types of cells would be diminished. Thus, by arguing that each DC displays only a limited number of such complexes, we only count those displayed at significant concentrations. This may be different for foreign peptide/MHCs, where displaying small numbers may be sufficient.

\section{HUMAN AND MOUSE MUTATIONS THAT LIMIT T-CELL PRODUCTION RESULT IN THE VAST MAJORITY OF PERIPHERAL CD4 T CELLS BEING MEMORY-PHENOTYPE}

When Tregs have too small a repertoire to efficiently inactivate DCs, stimulation of some of the conventional CD4 $\mathrm{T}$ cells an individual possesses can cause them to expand and differentiate. This concept very likely applies to a wide range of mutational states in which production of CD4 T cells, both conventional and Tregs, is impaired. The most striking example is provided by Omenn syndrome (Marrella et al. 2011). This disorder may occur in neonates with almost any mutation that would have caused severe combined immunodeficiency in other settings. Mutations affecting the mechanisms through which $\mathrm{T}$-cell receptor and B-cell receptor VDJ/VJ joining occurs, resulting in a severely limited output of T cells, are a particularly frequent cause of Omenn syndrome.

Shortly after birth, infants with such mutations develop severe autoinflammation associated with virtually all their CD4 T cells being in an activated/memory-phenotype state and with severe lymphocytic and eosinophilic infiltration. A potentially important component of the autoreactivity seen in Omenn syndrome is due to a failure of negative selection in the thymus because individuals with this syndrome have defects in thymic development, an absence or diminished number of medullary thymic epithelial cells (mTECs) and a severe deficiency in expression of AIRE. However, it seems unlikely that an AIRE deficiency can fully explain the autoimmunity/ autoinflammation of Omenn syndrome patients because the phenotype of humans with Omenn syndrome, who have a very limited CD4 T-cell repertoire, is far worse than that of individuals with APECED, which is due to a total deficiency in AIRE. The latter have normal TCR repertoire generation, although they have a defect in negative selection (Mathis and Benoist 2009).

Our view is that in addition to the autoreactivity due to the failure to negatively select conventional CD4 T cells specific for self-antigens, Omenn syndrome is characterized by a failure of Tregs, because of their very limited repertoires, to control the expansion of CD4 $\mathrm{T}$ cells.

\section{SELECTION AND RANDOM DISPLAY OF PEPTIDE/MHCS BY DCs FAVOR BROAD REPERTOIRES OF CONVENTIONAL NAÏVE CD4 T CELLS}

The survival of naïve peripheral CD4 T cells depends on their periodic subthreshold interaction with self-peptide/ 
MHCs on DCs. Thus, it is essential that such T cells be selfreactive to some degree. Such self-reactivity would appear to be inherently dangerous because the possibility would always exist that at least some of these T cells might mount tissue-damaging responses when they confront DCs expressing their cognate peptide/MHCs, particularly if an adjuvant has been supplied or if Tregs are not available. Indeed, we have already cited a potential example of such activation in the course of $T$. gondii infection (Oldenhove et al. 2009).

Why has this requirement of autoreactivity for CD4 Tcell survival been evolutionarily selected? It would seem essential to use the self-peptide/MHC as the selecting ligand during development, to eliminate useless or highly self-reactive T cells, but why use it as the survival ligand in the periphery? One possibility is that it helps to ensure that the repertoire of naïve CD4 T cells in the periphery remains broad, an essential feature if the individual is to be prepared to make responses to a full range of pathogenic microbes.

It has been argued that the cells leaving the thymus first enter a "recent thymic emigrant" (RTE) pool (Berzins et al. 1998; Dion et al. 2004). Only some RTEs may be incorporated into the pool of long-lived naïve CD4 T cells. Because only useful peripheral CD4 T cells should survive in the periphery, an obvious basis for such admission to the naïve pool would be the possession of some minimal affinity for self-peptide/MHCs displayed by peripheral DCs, sufficient to allow the T cell's survival and consistent with the $\mathrm{T}$ cell having potential reactivity with foreign peptide/MHCs. The levels of expression of selfpeptide/MHCs in the periphery might differ from those expressed by cTECs in the thymus, thus necessitating this second selection.

However, if all DCs presented all peptide/MHCs to a similar degree, then those naïve T cells whose T-cell receptors interacted most strongly with peptide/MHCs might outcompete those that interacted less efficiently and thus come to dominate the population. As a result, the repertoire of naïve CD4 T cells would be progressively narrowed once full DC-carrying capacity has been reached. By limiting the number of peptide/MHCs any individual DC presents, a greater opportunity would exist for T cells whose receptors interacted "weakly" with their selecting self-peptide/MHC to find DCs where there was little competition and thus to receive adequate survival signals. That is, niches would exist that serve as survival refuges for a very wide range of CD4 T cells. Moreover, as long as not all niches are fully occupied, a progressive increase of the peripheral repertoire and of its "quality" would be facilitated.

\section{WHAT ABOUT TOLERANCE?}

It has been suggested that distinction of self from nonself may not be an organizing principle of the immune system but rather the true organizing principle is distinguishing dangerous cells from benign cells. One basis for this idea is the Janeway/Matzinger dictum that responses only occur in the presence of agents that are detected by the growing number of microbial sensors our innate system possesses. Without such stimulation, no response ensues, either to self or foreign molecules. Thus, selfreactive $\mathrm{T}$ cells will not make responses under such conditions. However, based on the evidence we have reviewed, we should rather expect that when Tregs are absent or a strong adjuvant is used, had self/nonself discrimination not been established by other means, a massive self-reactive response would then occur. Indeed, expansion of some self-reactive cells does occur not only in lymphopenic setting but also in the context of infection. Presumably, this expansion is not sustained after the infectious agent has been controlled and thus may be of limited significance.

The fact that not one but several mechanisms have evolved to eliminate or control potentially self-reactive cells attests to the great importance of self-nonself discrimination, operating along with an inherent discrimination of pathogens as such. In general, such a plethora of mechanisms would hardly have been evolved and certainly not preserved if each did not offer real benefit to the individual and the population.

Among these mechanisms, in addition to control by Tregs, are clonal elimination within the thymus and clonal anergy in the periphery. We argue the clonal anergy is mediated by the process of reactivity tuning, which leads to adjustment of activation thresholds.

That the failure of clonal elimination has severe consequences is almost too well known to be stated here but for completeness we reiterate the consequences of AIRE deficiency. AIRE is expressed in mTECs where it controls transcription of many tissue-specific proteins in the thymus (Mathis and Benoist 2009). Individuals lacking AIRE develop APECED, a disorder characterized particularly by autoimmunity to endocrine organs (Villasenor et al. 2005). Similarly, mutational inactivation of Foxp3, the master regulatory factor of Tregs, leads to human IPEX, involving massive and quickly fatal self-responses due to the absence of Tregs (Bennett et al. 2001).

\section{PROPOSITION 3: TUNING REGULATES RESPONSIVENESS TO SELF-PEPTIDE/MHCS}

The concept that Tregs maintain DCs in an "off" state and that adjuvants allow DCs to achieve an "on" state even in the presence of Tregs can be valid only if other established mechanisms of self-tolerance, including clonal elimination-based self-tolerance and tuning, are operative. In the absence of such mechanisms, massive responses to self would occur when "adjuvants" are administered or in the course of inflammatory responses. The reason this does not happen is that T-cell activation is restricted, even in the presence of adjuvants, by the requirement that a cellular activation threshold must be exceeded. Negative selection that occurred in thymus eliminates cells with the highest binding affinity for self-peptide/MHCs. Peripheral interactions with DCs expressing the self-peptide/ MHCs are needed for survival but, except under unusual 
circumstances, such interactions do not cause activation. This is presumably because those interactions are below the activation threshold. Do cells that reach the periphery continue to be actively inhibited from responding to selfpeptide/MHCs? We argue that although there is clearly some "hard-wired" minimal stimulation strength below which activation cannot occur, stimulation in the periphery often, or perhaps generally, exceeds this minimum. Indeed some of the autoimmune potential of a fraction of the T-cell population is revealed under certain conditions, including lymphopenia, as we have discussed. To avoid autoimmunity, especially in the presence of adjuvants that "override" Treg-mediated control, the operative activation threshold needs to be constantly elevated through a mechanism we designated "tuning" (Grossman and Paul 1992). Tuning is mediated by the very same repetitive interactions with self-peptide/MHCs that the cells require to maintain viability. In particular, repetitive interactions of $\mathrm{T}$ cells with their selecting ligands, to which they are necessarily moderately autoreactive, leads the T cells to tune their TCR signaling apparatus so that the signals generated by such interactions are below the adjusted activation threshold of the cell. Such cells are anergic to their self-peptide/MHCs and generally remain so even when adjuvants are present or in lymphopenic situations. We should point out that these ideas would apply equally well if the T cells were interacting with peptide/ MHCs in which the peptides were derived from commensal microorganisms. We use "self-peptide/MHC" to represent complexes composed either of self or commensalderived peptides.

The tuning mechanism, although effective, is breached in some instances, as shown by those clones that expand in lymphopenic states in response to self-peptide/MHC stimulation or that "nonspecifically" expand during infection. We suggest that when conventional CD4 T cells are introduced into lymphopenic environments, lacking Tregs, those with the highest affinity for their self-peptide/MHCs will cause the activation of DCs bearing that complex and, in turn, these now activated ("on") DCs will cause a strong stimulation of the T cells, sufficient to exceed the tuned activation threshold. In the case of infection, the situation is generally similar. However, it is DCmediated pathogen sensing that aids in the T-cell-mediated turn "on" of those DCs, even in the presence of Tregs.

One may ask why the CD $4 \mathrm{~T}$ cells do not retune to the new level of DC-mediated stimulation rather than become activated. In our view, tuning is the cellular physiologic response to moderate and/or gradual changes in the level of signaling while strong, sudden perturbations result in activation because the cell will not have had the opportunity to gradually adjust its signaling pathways to set a new activation threshold (Grossman and Paul 2001). Although in the instances stated above the perturbation does not result from encountering cognate antigen and high-affinity binding, we suspect that activation is facilitated in these instances by striking increases in DC coreceptor and class II MHC expression. Normally, the tuning interaction of a CD4 T cell and a DC occurs with a relatively low level of expression of class II MHC molecules and coreceptors on the DC surface. It is even possible that the cell-surface expression by DCs of class II MHC, CD80 and CD86 is actively down-regulated, dynamically, during this recurrent interaction, as is the activation threshold of the T cells (Vendetti et al. 2000). The level of T-cell tuning, in turn, is adjusted to the controlled level of subthreshold stimulation by the DCs. However, when DCs are turned "on" by pathogens, or merely stimulated by adjuvants/inflammation in the "off" state, they upregulate MHC class II expression (and possibly other accessory molecules) and thus may more potently present more self-peptide/MHC to a tuned cell, stimulating it to a level exceeding the adjusted activation threshold established by interacting with a DC expressing relatively low levels of class II MHC molecules. As suggested, failure to quickly adapt the level of tuning to the new level of stimulation in such cases is due to the abrupt nature of the perturbation, but the "forbidden" responses generally do not last long.

Here we have raised the possibility that the tuning interaction of a CD4 T cell and a DC is a reciprocal process, dynamically adjusting the activation thresholds of both parties (Grossman and Paul 2000, 2001). Both conventional CD4 T cells and Tregs presumably participate in the proposed subthreshold interactions. In addition to the maintenance of tolerance, these interactions are proposed to regulate the ability of DCs to deliver viability and selfrenewal signals to the $T$ cells and help maintain their functional integrity. Because the interaction is effectively structured in terms of receptor specificities into many DCdefined niches, the process we have described provides a sound basis for homeostatic regulation, limiting clonal sizes and maintaining diversity.

\section{SUMMARY}

Here we have argued that a naïve CD4 T cell requires a potent innate stimulus to respond to a challenge with a cognate antigen. Although in most respects this is correct, it deals with the circumstance in which the antigen-presenting DC is inhibited by Tregs and by concomitant subthreshold interactions with conventional $\mathrm{CD}^{+} \mathrm{T}$ cells that recognize the self-peptide/MHCs through which they had been selected in the thymus.

Although the regulating cells, both Tregs and conventional CD4 $\mathrm{T}$ cells, need not individually recognize the same peptide/MHC that the regulated $\mathrm{T}$ cells recognize, they must recognize complexes displayed by the same DC. We propose that DCs each present effectively only a limited set of self-peptide/MHCs so a broad repertoire of Tregs is essential if autoimmunity is to be avoided. Similarly, narrow repertoires of conventional $\mathrm{T}$ cells may result in a less effective tonal inhibition of DCs and further increase the chance of autoimmune manifestations. The fundamental advantage gained by limiting the set of self-peptide/MHCs each individual DC presents and making that set random is that it helps to maintain broad repertoires of naïve CD4 $\mathrm{T}$ cells, because competition for survival is restrained. 
We argue that self-tolerance at the level of the T cells is essential to avoid damaging autoimmunity. Although distinguishing self from nonself may be of limited importance when DCs are under control by Tregs and by other T cells that keep them in the "off" state, T-cell tolerance to self-molecules becomes essential as soon as strong innate signals are introduced that help inducing foreign- and some self-antigen-presenting DCs into an "on state." Extensively studied tolerance mechanisms include clonal elimination in the thymus and clonal anergy and receptor tuning. We regard clonal anergy as a manifestation of tuning. Receptor tuning dynamically adjusts the cell's activation threshold so that the self-peptide/MHC that it sees in the periphery and that is responsible for its survival does not lead to a full-blown response, even when the DC is in an "on" state. It is mainly foreign peptide/MHCs, which exceed the activation threshold established by tuning, that lead to such a response, in the presence of pathogen sensing induced signals that concomitantly overcome the Treg-mediated inhibition.

Tolerance-maintaining processes are both hierarchical and overlapping. Negative selection substantially reduces the number of $\mathrm{T}$ cells having the potential of undergoing receptor activation in the periphery in response to selfpeptide/MHC stimulation. Dynamic tuning of T-cell responsiveness further reduces the probability that the more autoreactive cells that are selected become activated in that way. Tuning of T cells and DCs is a reciprocal and mutually-enhancing process depending on recurrent subthreshold interaction. Receptor activation is necessary but not sufficient for a full-activation followed by significant clonal expansion; the latter can be rapidly blocked, semispecifically, by Tregs. Treg-mediated blockade, in turn, is overcome by adjuvants. The bottom line is that all of the following - high-affinity recognition of a self-ligand (failure of selection), failure of tuning to properly adjust the T-cell activation threshold, and failure of Tregs to inhibit or reverse the subsequent activation of the selfantigen-presenting DCs-would need to have occurred for autoimmunity to be manifested. Thus, autoimmunity is rare if all the controls are in place. In a lymphopenic setting, in which Treg function is limited, a small proportion of memory and a still smaller proportion of naïve CD4 $\mathrm{T}$ cells do expand and adopt effector functions. We propose that the "incompleteness" of regulation by tuning under these circumstances indicates that DCs may also be subject, as are T cells, to responsiveness-tuning in the intact organism, jointly imposed by Tregs and multiclonal subsets of conventional cells and requiring diverse repertoires of both. When the number of T cells is small the disposition of DCs to activate and become activated increases. In particular, poorly-tuned DCs upregulate the expression of cell surface class II MHC molecules. Such upregulated expression may result, especially in the presence of adjuvant, in signals that exceed the adjusted T-cell activation threshold. In the intact organism, tuning counteracts fluctuations in adjuvant/innate signaling to DCs, but abrupt/strong perturbations associated with specific responses to pathogens can evoke transient "bystander" responses by some autoreactive $\mathrm{T}$ cells to their own se- lecting self-peptide/MHCs. Tuning serves as an additional tolerance mechanism, in concert with central tolerance and Tregs, but has also been implicated in other regulatory processes including homeostasis (Grossman and Paul 2000, 2001).

Our view thus incorporates key elements of immunity including innate pathogen-sensing, regulatory $\mathrm{T}$-cell function, self-recognition, central tolerance, and tuning into a unified hypothesis in which self-recognition governs the regulation of the immune system and restricts the consequences of pathogen sensing. We propose that this explanation of immune regulation being concurrently dependent on Pathogen Sensing, Regulatory T Cells, and Responsiveness-Tuning be designated the PaRT hypothesis.

\section{ACKNOWLEDGMENTS}

The research on which this work is based was supported by the National Institute of Allergy and Infectious Diseases Division of Intramural Research through Projects Z01-AI000493-27 and Z01-AI000926-13. We thank our many colleagues at NIH and throughout the scientific community for their generous exchange of ideas that have helped immeasurably in the development of the concepts described in this paper. We thank Shirley Starnes for expert editorial assistance.

\section{REFERENCES}

Almeida AR, Rocha B, Freitas AA, Tanchot C. 2005. Homeostasis of $\mathrm{T}$ cell numbers from thymus production to peripheral compartmentalization and the indexation of regulatory T cells. Sem Immunol 17: 239-249.

Azzam HS, Grinberg A, Lui K, Shen H, Shores EW, Love PE. 1998. CD5 expression is developmentally regulated by T cell receptor (TCR) signals and TCR avidity. J Exp Med 188: 2301-2311.

Bennett CL, Christie J, Ramsdell F, Brunkow ME, Ferguson PJ, Whitesell L, Kelly TE, Saulsbury FT, Chance PF, Ochs HD. 2001. The immune dysregulation, polyendocrinopathy, enteropathy, X-linked syndrome (IPEX) is caused by mutations of FOXP3. Nat Genet 27: 20-21.

Benson A, Murray S, Divakar P, Burnaevskiy N, Pifer R, Forman J, Yarovinsky F. 2012. Microbial infection-induced expansion of effector $\mathrm{T}$ cells overcomes the suppressive effects of regulatory $\mathrm{T}$ cells via an IL-2 deprivation mechanism. $J$ Immunol 188: 800-810.

Berzins SP, Boyd RL, Miller JF. 1998. The role of the thymus and recent thymic migrants in the maintenance of the adult peripheral lymphocyte pool. J Exp Med 187: 1839-1848.

Dion ML, Poulin JF, Bordi R, Sylvestre M, Corsini R, Kettaf N, Dalloul A, Boulassel MR, Debré P, Routy JP, et al. 2004. HIV infection rapidly induces and maintains a substantial suppression of thymocyte proliferation. Immunity 21: 757-768.

Freund J, Lipton MM, Thompson GE. 1953. Aspermatogenesis in the guinea pig induced by testicular tissue and adjuvants. $J$ Exp Med 97: 711-726.

Grossman Z, Paul WE. 1992. Adaptive cellular interactions in the immune system: The tunable activation threshold and the significance of subthreshold responses. Proc Natl Acad Sci 89: $10365-10369$.

Grossman Z, Paul WE. 2000. Self-tolerance: Context dependent tuning of $\mathrm{T}$ cell antigen recognition. Sem Immunol 12: 197-203. 
Grossman Z, Paul WE. 2001. Autoreactivity, dynamic tuning and selectivity. Curr Opin Immunol 13: 687-698.

Grossman Z, Min B, Meier-Schellersheim M, Paul WE. 2004 Concomitant regulation T-cell activation and homeostasis. Nat Rev Immunol 4: 387-395.

Hsieh CS, Liang Y, Tyznik AJ, Self SG, Liggitt D, Rudensky AY. 2004. Recognition of the peripheral self by naturally arising $\mathrm{CD} 25^{+} \mathrm{CD}^{+} \mathrm{T}$ cell receptors. Immunity 21: 267-277.

Janeway CA. 1989. Approaching the asymptote? Evolution and revolution in immunology. Cold Spring Harb Symp Quant Biol 54 (Pt 1): 1-13.

Kedl RM, Schaefer BC, Kappler JW, Marrack P. 2002. T cells down-modulate peptide-MHC complexes on APCs in vivo. Nat Immunol 3: 27-32.

Kim J, Lahl K, Hori S, Loddenkemper C, Chaudhry A, deRoos P, Rudensky A, Sparwasser T. 2009. Cutting edge: Depletion of Foxp $3^{+}$cells leads to induction of autoimmunity by specific ablation of regulatory $\mathrm{T}$ cells in genetically targeted mice. J Immunol 183: 7631-7634.

Mandl JN, Monteiro JP, Vrisekoop N, Germain RN. 2013. T cell-positive selection uses self-ligand binding strength to optimize repertoire recognition of foreign antigens. Immunity 38: $263-274$.

Marrella V, Maina V, Villa A. 2011. Omenn syndrome does not live by V(D)J recombination alone. Curr Opin Allergy Clin Immunol 11: 525-531.

Mathis D, Benoist C. 2009. Aire. Annu Rev Immunol 27: $287-$ 312.

Matzinger P. 1994. Tolerance, danger, and the extended family. Annu Rev Immunol 12: 991-1045.

Milner JD, Ward JM, Keane-Myers A, Paul WE. 2007. Lymphopenic mice reconstituted with limited repertoire T cells develop severe, multiorgan, Th2-associated inflammatory disease. Proc Natl Acad Sci 104: 576-581.
Min B, Foucras G, Meier-Schellersheim M, Paul WE. 2004. Spontaneous proliferation, a response of naïve CD4 T cells determined by the diversity of the memory cell repertoire. Proc Natl Acad Sci 101: 3874-3879.

Oldenhove G, Bouladoux N, Wohlfert EA, Hall JA, Chou D, Dos Santos L, O’Brien S, Blank R, Lamb E, Natarajan S, et al. 2009. Decrease of Foxp $3^{+}$Treg cell number and acquisition of effector cell phenotype during lethal infection. Immunity 31: $772-786$.

Pasare C, Medzhitov R. 2003. Toll pathway-dependent blockade of $\mathrm{CD} 4{ }^{+} \mathrm{CD} 25^{+} \mathrm{T}$ cell-mediated suppression by dendritic cells. Science 299: 1033-1036.

Quiel J, Caucheteux S, Laurence A, Singh NJ, Bocharov G, Ben-Sasson SZ, Grossman Z, Paul WE. 2011. Antigen-stimulated CD4 T-cell expansion is inversely and log-linearly related to precursor number. Proc Natl Acad Sci 108: 33123317.

Shevach EM. 2011. Biological functions of regulatory T cells. Adv Immunol 112: 137-176.

Singh NJ, Bando JK, Schwartz RH. 2012. Subsets of nonclonal neighboring $\mathrm{CD}^{+} \mathrm{T}$ cells specifically regulate the frequency of individual antigen-reactive $\mathrm{T}$ cells. Immunity 37: 735-746.

Vendetti S, Chai JG, Dyson J, Simpson E, Lombardi G, Lechler R. 2000. Anergic T cells inhibit the antigen-presenting function of dendritic cells. J Immunol 165: 1175-1181.

Villasenor J, Benoist C, Mathis D. 2005. AIRE and APECED: Molecular insights into an autoimmune disease. Immunol Rev 204: $156-164$

Younes SA, Punkosdy G, Caucheteux S, Chen T, Grossman Z, Paul WE. 2011. Memory phenotype CD4 T cells undergoing rapid, nonburst-like cytokine-driven proliferation can be distinguished from antigen-experienced memory cells. PLoS Biol 9: e1001171. 


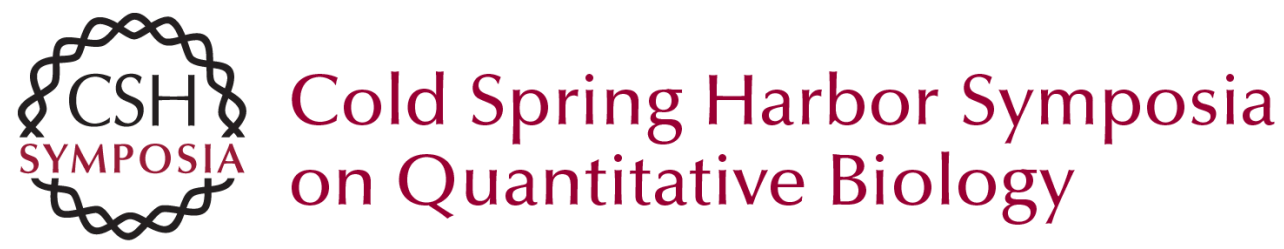

\title{
Pathogen-Sensing, Regulatory T Cells, and Responsiveness-Tuning Collectively Regulate Foreign- and Self-Antigen Mediated T-Cell Responses
}

\author{
William E. Paul, Joshua D. Milner and Zvi Grossman
}

Cold Spring Harb Symp Quant Biol 2013 78: 265-276 originally published online October 7, 2013 Access the most recent version at doi:10.1101/sqb.2013.78.020198

$\begin{array}{ll}\text { References } & \text { This article cites } 29 \text { articles, } 12 \text { of which can be accessed free at: } \\ \text { http://symposium.cshlp.org/content/78/265.full.html\#ref-list-1 }\end{array}$

\section{License}

Email Alerting Receive free email alerts when new articles cite this article - sign up in Service the box at the top right corner of the article or click here. 